\title{
An Improved Energy Aware Distributed Clustering Protocol for Wireless Sensor Networks
}

\author{
Vrinda Gupta \\ Department of Electronics and Communication Engineering, National Institute of Technology, Kurukshetra, Haryana- \\ 136119, India \\ E-mail: vrindag16@gmail.com \\ Rajoo Pandey \\ Department of Electronics and Communication Engineering, National Institute of Technology, Kurukshetra, Haryana- \\ 136119, India \\ E-mail: rajoo_pandey@nitkkr.ac.in
}

\begin{abstract}
The present work considers energy aware distributed clustering protocol (EADC) which is commonly employed for balancing the energy consumption in non-uniformly deployed sensor networks. In the EADC, residual energy is considered as the primary clustering parameter and the clusters are constructed using uniform competition radius. However in non-uniform node distribution scenarios, more energy imbalance occurs in the network due to variation in intracluster energy consumption. For counter balancing it, an inter-cluster energy-efficient multi-hop routing protocol is utilized. In the EADC protocol, cluster heads select the relay node based on residual energy and cluster member count information. But this approach is less efficient in energy balancing. In this paper, an improved energy aware distributed equal clustering protocol is projected. Our scheme considers the relay metric directly in terms of energy expense in relaying the sensed data to the base station taking into account not only the residual energy and member count information but also distance information. It provides better balancing of energy in comparison with the existing approach. Three different scenarios created by varying node distribution are used for evaluating the performance of the proposed protocol and comparison is made with the EADC protocol. The results show that the proposed scheme extends network lifetime in all the scenarios.
\end{abstract}

Index Terms - Clustering, EADC, Multi-hop routing, Network lifetime, Wireless sensor networks.

\section{INTRODUCTION}

Wireless sensor networks usually are designed for harsh environment applications where human intervention is not possible such as forest fire, wild life monitoring, tunnels, bridges, coal mines etc. to name a few. Such data gathering applications require sensors to be deployed in large numbers and the data to be sensed from all locations. However, these sensor nodes are energy limited. In many applications the base station (BS) is usually placed far away from the sensing field and the data is gathered periodically by the BS. In order to address the energy constraint, large amount of research work has been carried out in the last decade [1-3]. For such continuous monitoring networks, clustering with hierarchical topology is successful [4-7]. It is exhibited that clustering the network offers greater lifespan with an increase of about 2-3 times than the network with direct data transmission [8].

There are many other advantages of using clustering protocols in data-gathering networks. As dense networks involve large volume of traffic among the sensors, it leads to interference problems. In such scenarios, grouping the sensors is extremely beneficial. Further, it minimizes the number of long distance transmissions and results into saving of the energy. In clustering, cluster heads $(\mathrm{CHs})$ coordinate the activities of its member nodes and the normal sensor nodes (cluster members) remain in sleep mode, which further leads to energy saving [9]. This is possible because CHs execute TDMA scheduling for its member nodes [7, 10]. Also clustering facilitates data aggregation at cluster head. Thereby the number of data transmissions further minimizes, and the network lifetime prolongs [9].

The data transmission in clustering protocols occurs in two steps, one is within the clusters i.e. intra-cluster and another is between the clusters and the BS i.e. intercluster. In addition, the communication in a wireless sensor network clustering protocol can be taken up either by employing direct transmission through single hop, or using multi-hop routing $[11,12]$. For data transmissions within the cluster i.e. from member nodes to $\mathrm{CH}$, most of the clustering protocols use single hop communication, as the transmission distance is relatively short e.g. LEACH [7], LEACH-DT [10], HEED [13] etc. Multi-hop communication between the sensor nodes and the cluster head is promoted when the propagation loss exponent is high as in buildings, factories, or dense vegetation regions [1]. Direct transmission also has its benefits in saving of energy as the radio dissipates energy in not only transmission but also in reception. But it is used only when the transmission distance is within certain threshold 
distance only [14]. This is, because the energy expense increases according to the fourth power of the distance $[10,15]$. There are number of clustering protocols developed which propose multi-hop communication for achieving more energy-efficient inter-cluster communication viz. Multi-hop LEACH [16], EADC [17], EDUC [18].

Many research works has defined the network lifetime to be when first node is dead (FND). The idea behind this assumption is that it is important that all the nodes of the network die out approximately at the same time in order to avoid early loss of sensing coverage, and likely partitioning of the network [7, 10, 13]. But, as the lifetime requirement is application-specific, considering first node dead as the lifetime definition is not a generic one [19]. There are different types of sensor network's applications [20] and therefore it is better to evaluate the lifetime of the network at different stages viz. the time when certain percentage of nodes fail [21].

In wireless sensor networks, one of the primary concerns is maximization of network lifetime because after the network becomes dysfunctional, significant amount of energy should not remain in the nodes, otherwise it is wasted. In a clustering protocol, a $\mathrm{CH}$ is heavily burdened as it is responsible for execution of various tasks such as cluster formation, data aggregation, data transmission and relaying. Cluster heads therefore consume more energy as compared to non- $\mathrm{CH}$ nodes. For single hop communication, cluster heads which are far away from BS drain out their energy primarily because of the long distance transmission. But when using multi-hop communication in clustering protocols, then, the cluster heads near the base station deplete their energy quickly because of the extra burden of traffic relaying. This unbalanced communication load results in energy hole or hot spot area. Due to this, data required from all corners may not be obtained and the network performance is ultimately affected. Thus, the energy consumption among all the network nodes must be balanced. In clustered networks, therefore, there is inevitable problem of energy imbalance among sensor nodes [18]. Recently, lot of research has been carried out to address energy imbalance and mitigate energy hole problem for clustered WSNs. A number of strategies such as using node mobility [22, 23]; mobile sink [24-27]; hierarchical deployment [28]; nonuniform clustering [18,29]; data compression and traffic aggregation [27, 30]; node distribution [22, 31, 32]; etc. have been proposed for solving energy imbalance problem.

In this paper, an attempt has been made to improve network lifespan of an energy-aware distributed clustering protocol EADC used in continuous monitoring applications [29]. The EADC employs uniform clustering algorithm to mitigate the energy imbalance. The key idea of proposed scheme is during selection procedure of traffic relaying. The energy cost involved in relaying is directly considered as the metric for selecting one of the feasible nodes as a relay node instead of only residual energy and the cluster member count information used in the EADC. The performance of the proposed protocol is compared with the existing energy aware distributed clustering protocol. The proposed scheme poises the energy consumption of the nodes in the network for uniform distribution as well as for non-uniform distribution.

The remainder of this paper is organized as follows: Section 2 reviews the related work and Section 3 presents the network model. Section 4 describes the proposed protocol operation in detail and Section 5 analyzes the protocol characteristics. Section 6 gives the simulation results of our sensor deployment schemes and compares it with existing protocols. Finally Section 7 concludes the paper.

\section{RELATED WORK}

Earlier research work undertaken in the area of clustering algorithms has been primarily based on the rotation of role of cluster heads in every round, and selecting cluster heads with more residual energy in order to enhance the lifespan of the network. A pioneer protocol, low-energy adaptive clustering hierarchy (LEACH) protocol [15] and its many variants such as LEACH-DT [10] or a multi-hop variant of LEACH, called as M-LEACH [1] have been developed in the past. The LEACH protocol assumes one-hop communication between the nodes and to the base station. This makes it unsuitable for large-scale networks. Also the $\mathrm{CH}$ selection is probabilistic without even considering the residual energy of the nodes. A hybrid energy-efficient distributed (HEED) clustering algorithm is proposed in [13], which select cluster head according to not only the node residual energy but also intra-cluster communication costs. It uses multi-hop communication among the cluster heads for inter-cluster communication. It is successful in prolonging the network lifetime but not so effective in balancing the communication load as node's closer to BS still die faster. Another protocol available is distributed energy efficient clustering algorithm (DEEC) [33], wherein cluster heads are chosen by a probability which is based on the ratio of residual energy of a node and the average energy of the network [33]. In all these energy-efficient clustering schemes, although periodic rotation of cluster head function sees that nodes runs through energy more evenly, but it is not effective in solving the energy consumption balancing issue of many-to-one data gathering wireless sensor networks.

In the last few years, researchers have explored the strategies to extenuate the energy imbalance problem in hierarchical (cluster-based) WSNs. Many protocols have been developed which addresses this issue using nonuniform clustering technique [34]. They all attempt to organize the nodes into clusters of unequal size with the clusters nearer to BS to be of small size than those that are more distant from the BS in order to relieve the nodes of the extra burden of traffic relaying. Our focus in this paper is on equalizing the energy consumption in the network through uniform clustering only. 
Also there are many published works which utilize equal size clustering to balance the energy consumption in sensor networks. A balanced clustering algorithm (BCA) is promoted for irregularly deployed sensor networks in [35], which uses equal size clustering to improve energy efficiency and balance energy consumption in non-uniformly deployed sensor networks. The cluster head selection is based on the node density parameter. It attempts to minimize redundant sensing and transmissions. By using the sensing population information, $\mathrm{CHs}$ turn the redundant nodes into sleep mode, and results in prolonging the network lifetime.

In [36], the authors proposed an energy efficient routing algorithm (ERA), which conserves energy during cluster set up phase and also facilitates data routing through maintaining a virtual backbone of $\mathrm{CHs}$. The selection of $\mathrm{CHs}$ is based on residual energy and the clusters are formed with non-cluster heads nodes joining the $\mathrm{CH}$ based on residual energy and distance to the BS. Further in it, relaying load of the $\mathrm{CHs}$ is balanced with their residual energy. For evaluation, random and grid deployment scenarios have been considered and performance metrics used are network life time, energy consumption, power imbalance factor, and data aggregation.

Another article [37], forms clusters of even sizes to equalize energy consumption among nodes. The authors in this propose a decentralized energy efficient hierarchical cluster based routing algorithm. For cluster head selection, the parameters considered are energy consumption, the actual distance the data traverses to reach the $\mathrm{BS}$, and the adjustment degree of adjusting the intra-cluster and inter-cluster energy consumption. It controls the number of control message exchanges while constructing routing tree and thereby saves energy.

\section{PRELIMINARIES}

This section presents the network model, the energy model and the data aggregation model used in the paper.

\section{A. Network model}

The network comprises of randomly deployed $N$ number of sensor nodes in an $M$ x $M$ area. The nodes are energy heterogeneous i.e. different initial energy is assigned to the sensor nodes. The BS is far away from the sensor field and its location is assumed to be known to each node. The nodes use power control to adjust the transmission power depending on the transmission distance. The nodes are not location aware. The cluster heads can transmit their data directly with BS depending on their distance to the BS.

\section{B. Energy model}

The transmitter consumes energy in running the radio electronics circuitry and the transmit amplifier circuitry, whereas, the receiver's energy consumption is only in radio electronics part [7, 15]. Also, depending on the transmission distance, both the free space $\varepsilon_{f_{s}}$ and multipath fading $\varepsilon_{m p}$ channel models are used. If the distance is less than a threshold level, the free space model is used; otherwise the multipath model is used. When transmitting the $l$-bit data to a distance $d$, the radio expends according to (1).

$$
\begin{array}{r}
E_{T X}(l, d)=E_{T X-e l e c}(l)+E_{T X-a m p}(l, d) \\
=\left\{\begin{array}{l}
l * E_{\text {elec }}+l * \varepsilon_{f s} * d^{2}, d<d_{t h} \\
l * E_{\text {elec }}+l * \varepsilon_{m p} * d^{4}, d \geq d_{t h}
\end{array}\right.
\end{array}
$$

When receiving the 1-bit data, the radio expends according to (2).

$$
\begin{aligned}
E_{R X}(l) & =E_{R X-\text { elec }}(l) \\
& =l E_{\text {elec }}
\end{aligned}
$$

\section{Data aggregation model}

In the present work, the infinite compressibility model is used for data aggregation [4, 15]. It is assumed that cluster head collect the data from its member nodes and aggregate it into a single packet of fixed length irrespective of the number of received packets.

\section{THE IMPROVED EADC PROTOCOL MECHANISM}

The clustering method used is similar in operation to EADC protocol [17]. After deployment, the nodes first compute its distance from BS. The signal broadcasted by $\mathrm{BS}$ is listened by all nodes. On the basis of the received signal strength, each node approximates its distance to BS The performance of the protocol is tested in terms of number of rounds it can deliver the sensed data to the BS. Each round comprises of set-up phase in which cluster is formed and steady state phase in which data transmission takes place. The set-up phase is further sub-divided into three sub-phases of durations $T_{1}, T_{2}$ and $T_{3}$ respectively. During $T_{1}$, each node broadcasts a Node_Msg, which contains its residual energy along with its id. All the nodes which are in its radio range, receive the Node_Msg from all its neighbors. Each node then works out the average residual energy, Eavg_res of the cluster according to (3).

$$
E_{a v g_{-} r e s}=\left(\sum_{j=1}^{m} s_{j} \cdot E_{r}\right) / n b
$$

where $s_{j}$ is one of the node, $s_{j} . E r$ is the residual energy of $s_{j}$, and $n b$ is the number of neighbors. After $T_{l}$ has timed out, the next sub-phase i.e. cluster head competition phase of duration $T_{2}$ commences. In this subphase cluster heads are elected. Each node calculates its wait time for broadcasting the Head_Msg according to (4). Here, $t$ represents the delay time of a node to compete for being cluster head. 


$$
t= \begin{cases}\frac{E_{\text {avg_res }}}{E_{r}} T_{2} V_{r}, & E_{r} \geq E_{\text {avg_res }} \\ E_{r}<E_{\text {avg_res }}\end{cases}
$$

where $E_{r}$ is the current residual energy of the node; $V_{r}$ is a real value randomly distributed in the interval $[0.9,1]$, which is used to reduce the probability that two nodes send Head_Msg at the same time [17]. If any node does not receive any Head_Msg, it broadcasts a Head_Msg within competition range $R_{c}$, advertising itself to be cluster head. The improved EADC scheme uses the same competition range to produce equal size clusters.

After cluster head competition sub-phase, wherein cluster heads are chosen, cluster formation sub-phase of duration $T_{3}$ commences. In this sub-phase, the non cluster head nodes join the nearest cluster head. These noncluster head nodes send the Join_Msg and the corresponding cluster head receives the Join request and forms the clusters. In the Join_Msg, the node gives its id and its remaining energy. The cluster head then prepares and broadcasts a TDMA Schedule_Msg containing the information of cluster member's data transmission. Thus each member node can remain in sleep mode until their time of data transmission. The sensor nodes energy consumption thus reduces by turning off their radios.

After the network is setup as clusters, steady state phase begins. In this phase, data transmission takes place. The data transmission occurs in two steps. In step 1, the member nodes transmit their sensed data according to the schedule to their respective cluster heads. This transmission is single hop and is known as intra-cluster communication. The cluster head after receiving the sensed data from its member nodes, computes the aggregated data. As the data sensed by cluster members is correlated, the incoming data is aggregated into one packet and stored by the $\mathrm{CH}$ node. This task of intracluster communication is carried out in all the clusters of the network.

The second step of data transmission is called intercluster communication. The cluster heads can transmit the data packet to the BS either directly, or through relaying. This decision is based on the distance of the $\mathrm{CH}$ to the BS. If the distance of a cluster head to the BS is less than threshold distance (dist_th), then direct transmission is executed, otherwise the $\mathrm{CH}$ communicates through other $\mathrm{CHs}$ which acts as relay node. For inter-cluster communication, the selection of next hop node (relay) is crucial.

In original EADC protocol, for relay node selection, each $\mathrm{CH} \mathrm{s} \mathrm{s}_{\mathrm{i}}$ computes parameter relay using (5) to choose $\mathrm{s}_{\mathrm{j}}$ as its next hop node and broadcasts its Route_Msg.

$$
\text { relay }=\alpha \frac{E_{j r}}{E_{\max }}+(1-\alpha) \frac{1}{s_{j} \cdot \text { mem }}
$$

where $E_{j r}$ is the residual energy of the node and $E_{\max }$ is the maximum value of initial energy of the nodes of the network. $s_{j}$.mem represents the member count of the $\mathrm{CH}$ node and $\alpha$ is a real value between 0 and 1 . Here, the node having maximum value of relay gets selected as relay node for forwarding the data to the BS. This implies that the $\mathrm{CH}$ node which has higher residual energy and lower member count will be selected as the relay node. Here, $s_{j}$ is a $\mathrm{CH}$ node which acts a relay node for $s_{i}$ which is not further away from BS than $s_{i}$ is from BS.

In our proposed scheme, the relay node selection is based on the energy estimate of each possible relay node. It will be more appropriate to select the relay node based on the actual energy cost rather than based on only the residual energy and count information used in (5). One of the feasible nodes is finally selected as the relay node according to the expression given in (6). In the proposed scheme, for inter-cluster communication process, each cluster head first broadcasts a message comprising of its node id, residual energy, member count, and distance to the BS. The $\mathrm{CH} s_{i}$ would choose $\mathrm{CH} s_{j}$ as relay node, if its remaining energy is largest value, after incorporating its intra-cluster energy consumption cost; inter-cluster transmission cost, and the cost of relaying the data from $s_{j}$ to $\mathrm{BS}$, where relay is computed as given in (6).

$$
\frac{s_{j} \cdot E_{j r}-s_{j} \cdot m e m * E_{R X} * D M-\left(s_{j} \cdot m e m+1\right) * E_{D A} * D M-E_{T X} * D M}{E_{\max }}
$$

where $s_{j} E_{j r}$ represents the current residual energy of $j^{\text {th }}$ node; mem represents the member count of $j^{\text {th }}$ node; $E_{R X}$ represents the energy cost in receiving the data from its members with packet length $D M ; E_{D A}$ represents the energy cost in aggregating the received data and $E_{T X}$ is the energy cost of transmitting the data packet from $\mathrm{CH} s_{i}$ to $\mathrm{CH} s_{j}$ and finally to BS. $E_{\max }$ is the maximum value of residual energy initially available in the network. $\mathrm{CH} s_{j}$ transmits the message directly to BS in case when $\mathrm{s}_{\mathrm{j}}$ is within the pre-determined dist_th, or when no other $\mathrm{CH}$ node is available to route the packet. Thus, $s_{i}$ selects the cluster head as relay node having the maximum value of relay i.e. one which has highest remaining energy for forwarding the data packet to the BS. With choosing a relay node in this way helps in achieving the energy balance and in extending the network lifespan.

\section{PROTOCOL ANALYSIS}

In this section, the proposed protocol is analyzed. The main characteristics of the proposed protocol are elaborated. It shows that the cluster heads are distributed evenly and energy consumption among cluster member nodes is balanced. Further it shows that the proposed protocol is energy efficient. The properties of the improved EADC protocol are as follows.

- The cluster heads elected are based on the ratio of the average residual energy and the remaining energy of the nodes given in (4). This helps in 
prolonging the network lifetime as the nodes having more remaining energy are selected.

- The cluster heads set elected covers the whole network. As in (4), $V_{r}$ parameter ensures that for any case of remaining energy of nodes, the wait time is less than or equal to duration $T_{2}$ of cluster head competition sub-phase. Therefore, any node can become a cluster head before the timer expires. Further, in case any node has not received the Head_Msg, it advertises itself to be cluster head.

- The relay metric used is defined directly in terms of energy, so it helps in prolonging the lifetime by selecting the route more efficiently for sending the data to the BS.

- The number of control messages transmitted is $N$ number of Node_Msg, $(N-k)$ number of Join_Msg, $k$ number of Head_Msg, Schedule_Msg and Route_Msg, if $k$ is the number of $\mathrm{CHs}$ selected in a round. Therefore the total number of control messages generated in a round in our improved EADC protocol comes out to be $N+(N-k)+k+k+$ $k=2 N+2 k$. Thus the control messages overhead is $\mathrm{O}(N)$.

\section{PERFormance EVAluAtion}

In this section, the performance of the improved EADC protocol is evaluated through simulation. The simulation was performed in MATLAB. In simulation the widely accepted simple radio model [7] for radio hardware energy dissipation has been used. The topology of the network, the simulation parameter used, and the simulation results obtained are presented in the subsequent sections. The cluster head distribution, the network remaining energy, and the network lifetime have been analyzed. The performance of the proposed protocol is compared with the existing EADC protocol [17] in order to demonstrate the improvement obtained in network lifetime.

\section{A. Simulation Environment}

Three scenarios were chosen for simulations:

Scenario 1: 100 nodes are uniformly deployed over an area of $200 \times 200 \mathrm{~m}^{2}$ shown in Fig 1(a).

Scenario 2: 100 nodes are non-uniformly deployed with more number of sensor nodes grouped together in the region towards the right side of sensor field i.e. near to BS, over an area of $200 \times 200 \mathrm{~m}^{2}$ as shown in Fig 1(b).

Scenario 3: 100 nodes are non-uniformly deployed with more number of sensor nodes grouped together in the region towards the left side of sensor field i.e. far from BS, over an area of $200 \times 200 \mathrm{~m}^{2}$ as shown in Fig $1(\mathrm{c})$.

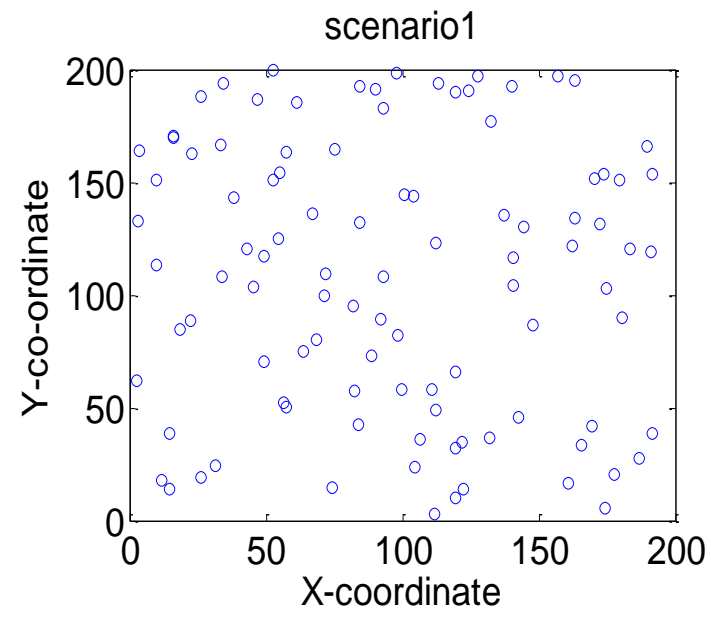

Fig.1(a). Network Topology of Scenario 1

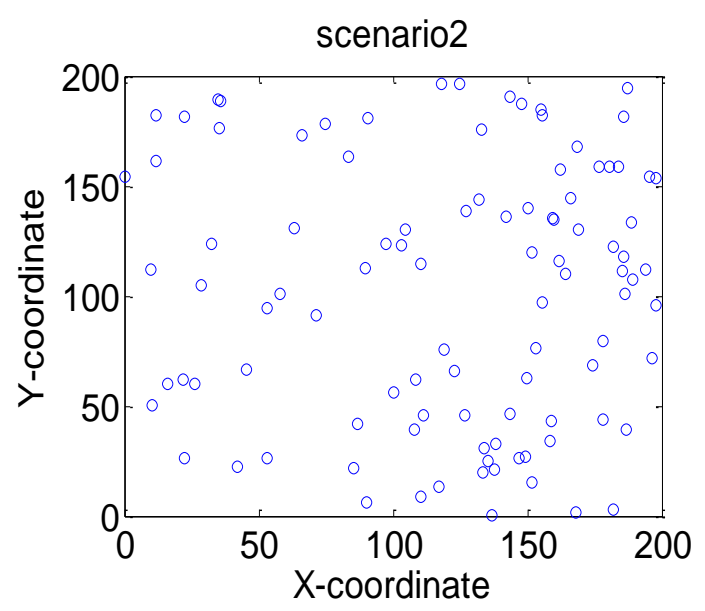

Fig.1(b). Network Topology of Scenario 2

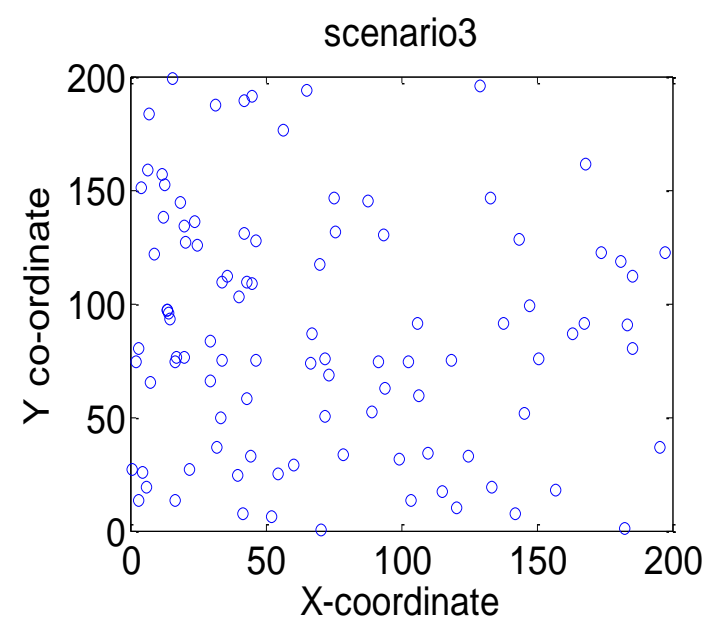

Fig.1(c). Network Topology of Scenario 3

\section{B. Simulation Parameters}

The parameters of the simulation used in the present paper are listed in Table I. 
Table 1. Simulation Parameters

\begin{tabular}{|l|l|l|}
\hline S.No. & Parameter & Value \\
\hline 1. & Network area & $200 \mathrm{~m} \mathrm{x} 200 \mathrm{~m}$ \\
\hline 2. & Base station location & $(250,100)$ \\
\hline 3. & No. of nodes & 100 \\
\hline 4. & Initial energy of nodes & $0.5-1.5 \mathrm{~J}$ \\
\hline 5. & Data packet size & $500 \mathrm{bytes}$ \\
\hline 6. & E elec & $50 \mathrm{~nJ} / \mathrm{bit}$ \\
\hline 7. & $\varepsilon_{\mathrm{fs}}$ & $10 \mathrm{pJ} / \mathrm{bit} / \mathrm{m}^{2}$ \\
\hline 8. & $\varepsilon_{\mathrm{mp}}$ & $0.0013 \mathrm{pJ} / \mathrm{bit} / \mathrm{m}^{4}$ \\
\hline 9. & $E_{D A}$ & $5 \mathrm{~nJ} / \mathrm{bit} / \mathrm{signal}$ \\
\hline 10. & $R_{c}$ & $90 \mathrm{~m}$ \\
\hline 11. & Threshold distance & $87.7 \mathrm{~m}$ \\
\hline 12. & $\alpha$ & 0.5 \\
\hline
\end{tabular}

\section{Results and Discussions}

In the simulation experiments, the energy model and the data aggregation model used is as described in section III. The results of the simulations are average of the several experiments performed. The following key metrics are used for studying the performance of the proposed protocol, such as:

- Number of cluster heads: This metric lays out the effect of node distribution in each scenario.

- Average energy consumption per round: This metric stands for the average energy consumption by all the nodes of the network in one round.

- Network remaining energy: This metric represents the total remaining energy of the network with respect to rounds.

- Network lifetime-FND: This metric is measured in terms of data collection rounds and represents the time when first node in the network dies.

- Network lifetime-PND: This metric corresponds to the time period from the instant the network starts functioning to the instant when 10 percent of the nodes are dead.

- Number of alive nodes: This metric shows the number of nodes which are alive with respect to rounds.

\section{a) $\mathrm{CH}$ distribution evaluation:}

Fig. 2 shows the average number of cluster heads generated in each scenario with the value of competition radius set as $90 \mathrm{~m}$. The figure illustrates that cluster head distribution is not affected with the node distribution. Further, it is observed that the number of cluster heads is controlled and distributed. In case of each scenario, two, three, four or five number of cluster heads is produced. This is because $R_{c}$ ensures that not more than one cluster head is produced in one cluster heads' coverage range. The clusters formed are equal size and this makes energy balance in the network.

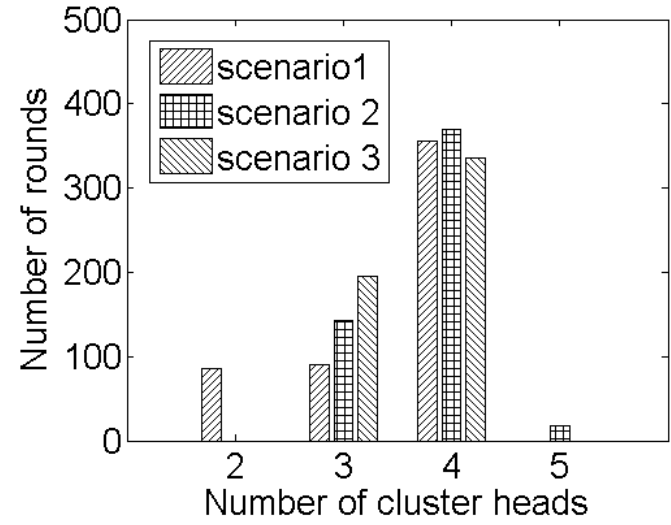

Fig.2. Average Number of Cluster Heads.

\section{b) Energy consumption evaluation}

The average energy consumption of the EADC and improved EADC protocol is evaluated for the three scenarios considered here. Fig. 3 shows average energy consumption per round in the network when each protocol is run until its lifetime. Energy consumption of a round comprises of the energy consumed during clustering topology formation and data transmission. It is observed that the mean energy consumption in our improved EADC protocol is slightly less than that in case of EADC protocol.

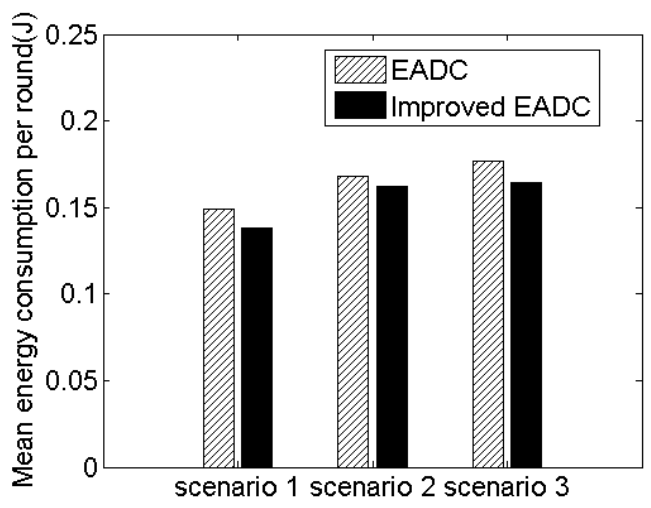

Fig.3. Average Energy Consumption of the Sensor Network.

The improved EADC protocol balances load among cluster heads by selecting the relay node taking into consideration the energy, member count and distance parameters. This can result in energy saving and enhancement of network lifetime.

\section{c) Network remaining energy evaluation}

Fig. 4 shows the total remaining energy of the network in improved EADC protocol with respect to number of rounds in each scenario. It is observed that residual energy of nodes in the network reduces at almost the same rate initially in all the scenarios. In later rounds, the difference can be seen in the node's remaining energy in different scenarios. The remaining energy depletes more quickly in scenario 3 , then in scenario 2 and lastly in scenario 1. In scenario 2, cluster heads in dense area has more member count, while in scenario 3 , cluster heads 
selected as relay node would require covering larger distance to transmit the data packet to $\mathrm{BS}$, therefore in these scenarios, energy depletes faster than uniformly distributed scenario i.e.scenario1.

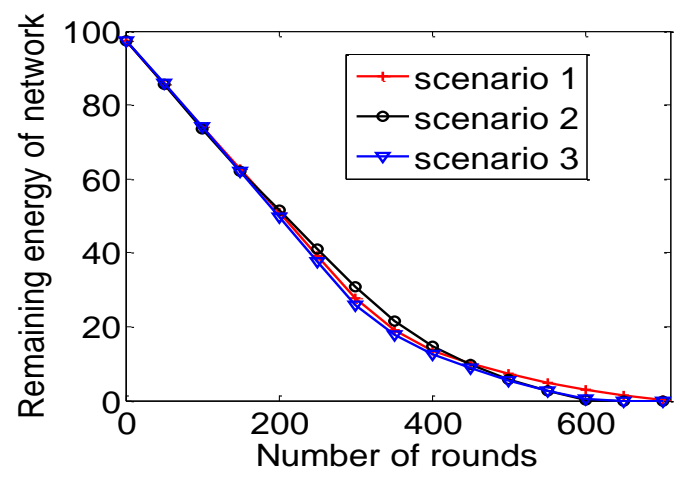

Fig.4. Average Residual Energy of Network.

\section{d) Network lifetime evaluation}

The network lifetime is evaluated here in two ways. One evaluation is based on the round when first node dies (FND) and another measurement is the round when 90 percent of the nodes are alive (PNA). The original EADC and improved EADC protocols are run in the three scenarios. As shown in Figs. 5, and 6, there is improvement in network lifespan, FND and PNA of improved EADC in each scenario compared to EADC protocol. The improved EADC protocol enhances the network lifetime when FND is the metric by $12 \%, 180 \%$ and $2 \%$ respectively and when PNA is the metric, by $7 \%$, $5 \%$ and $4 \%$ respectively for scenarios 1,2 and 3 as compared to EADC protocol. The network lifetime in improved EADC gets enhanced because of the energy efficient relaying methodology adapted. As the improved EADC protocol does relaying directly based on the energy estimate, and the ratio of intra-cluster \& intercluster energy consumption gets balanced in the proposed protocol. Therefore, the protocol is successful in extending the lifetime in case of non-uniform scenarios.

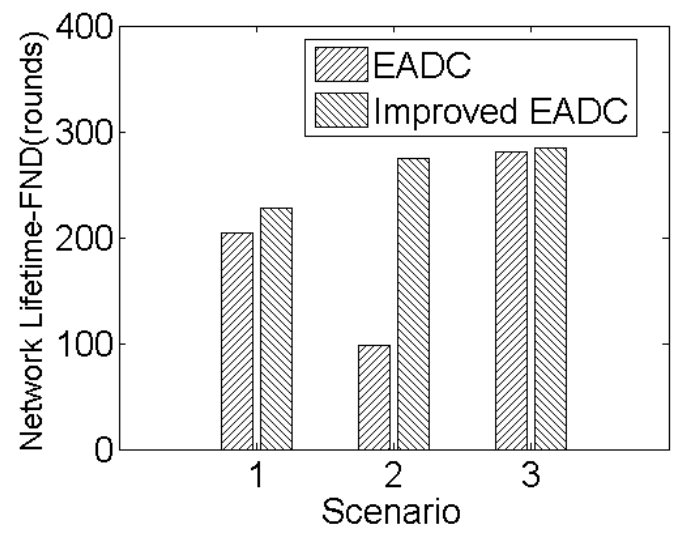

Fig.5. Network Lifespan when first Node Dies.

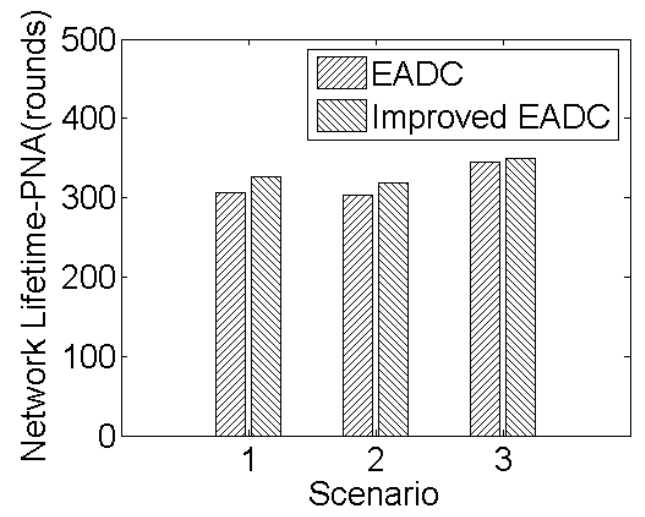

Fig.6. Network Lifespan when 90 Percent Nodes are Alive.

\section{e) Number of alive nodes evaluation}

The improvement gained through our improved EADC protocol is further laid out in each of the three scenarios. Fig. 7, 8 and 9 respectively shows the number of alive nodes with respect to rounds in case of scenario 1, 2 and 3. As observed, the improved EADC protocol achieves better energy efficiency and balancing than the EADC protocol. From the results, it can be concluded that the improved EADC protocol is able to address the nonuniform distribution and energy balance problem successfully.

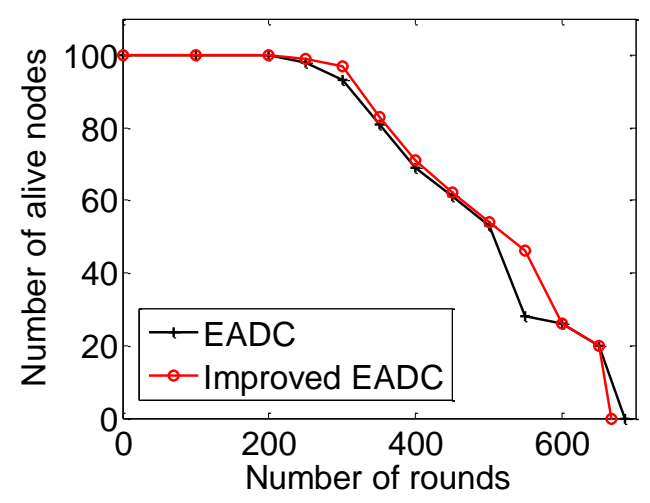

Fig.7. Number of Alive Nodes for Scenario 1.

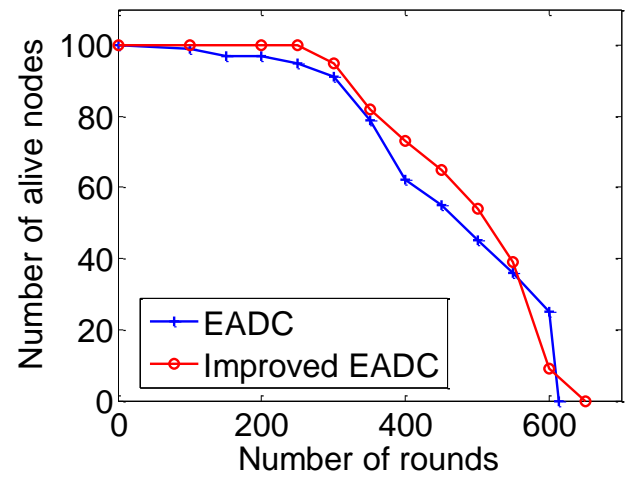

Fig.8. Number of Alive Nodes Rounds for Scenario 2. 


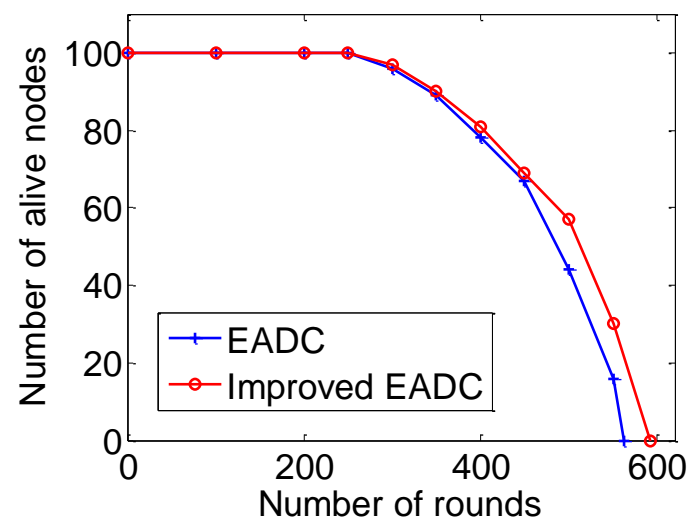

Fig.9. Number of Alive Nodes for Scenario 3.

\section{CONCLUSIONS}

In this paper, an energy aware distributed clustering protocol (EADC) has been extended in order to improve the lifespan of WSN. The uniform clustering technique is used in this work. The relay node selection procedure for forwarding the data towards the BS is based directly in terms of energy estimate. The simulation results show that network lifespan is extended effectively in each scenario compared to the EADC protocol. This improvement shows the effectiveness of the proposed improved EADC protocol in terms of balancing the energy and distributing the clusters in both uniform and non-uniform scenarios.

\section{REFERENCES}

[1] V. Mhatre, C. Rosenberg, Homogeneous vs. heterogeneous clustered networks: a comparative study, in: Proceedings of IEEE, ICC, vol.6, 2004, pp.3646-3651.

[2] M.H. Awaad, W.A. Jebbar, Extending the WSN lifetime by dividing the network area into a specific zones, Intl. Journal Computer Network and Information Security, 2015, 2, 33-39.

[3] X. Gu, J. Yu, D. Yu, G. Wang, Y. Lv, ECDC: an energy and coverage-aware distributed clustering protocol for wireless sensor networks, Computers and Electrical Engineering 40 (2014) 384 - 398.

[4] S. Bandyopadhyay, E. J. Coyle, An energy efficient hierarchical clustering algorithm for wireless sensor networks, in: IEEE INFOCOM, 2003, 1713-1723.

[5] M. Ye, C. Li, G. Chen, J. Wu, EECS: An energy efficient clustering scheme in wireless sensor networks, IEEE International Conference on Performance, Computing, and Communications, 2005, pp. 535-540.

[6] V. Gupta, R. Pandey, Research on energy balance in hierarchical clustering protocol architecture for WSN, IEEE Intl. Conf. on Parallel, distributed and Grid Computing, 2014, 115-119.

[7] W.B. Heinzelman, A. Chandrakasan, H. Balakrishnan, Energy efficient communication protocol for wireless microsensor networks, in: Proc. 33rd Hawaii Intl Conference on System sciences (HICSS'00), 2000, pp. 8020-8029.

[8] A. F. Liu, W. X. You, C. Z. Gang, G. W. Hua, Research on the energy hole problem based on unequal cluster- radius for wireless sensor networks, Computer Communications 33 (2010) 302-321.

[9] N. Vlajic, D. Xia, Wireless Sensor Networks: To cluster or not to cluster?, in: Proc. of the International Symposium on a World of Wireless, Mobile and Multimedia networks, 2006.

[10] S. H. Kang, T. Nguyen, Distance based Thresholds for cluster head selection in wireless sensor networks, IEEE Communication Letters, 16(9) (2012) 1396-1399.

[11] S. Verma, Prachi, Communication architecture for underwater wireless sensor network, Intl. J. Computer Network and Information Security, 2015, 6, 67-74.

[12] M. Perillo, Z. Cheng, W. Heinzelman, On the problem of unbalanced load distribution in wireless sensor networks, in: Proc. of IEEE GLOBECOM Workshops on Wireless Ad hoc and sensor networks, Dallas, TX, 2004, pp.74-79.

[13] O. Younis, S. Fahmy, HEED: a hybrid, energy-efficient, distributed clustering approach for ad hoc sensor networks, IEEE Trans. Mobile Comput. 3(4) (2004), 366-379.

[14] B. Tavli, Energy-efficient relaying in wireless networks, International Journal Electron. Commun. (AEÜ) 63 (2009) 695-698.

[15] W.B. Heinzelman, A. Chandrakasan, H. Balakrishnan, An application-specific protocol architecture for wireless microsensor networks, IEEE Transactions on Wireless Communications 1(4) (2002) 660-670.

[16] F. Xiangning, S. Yulin, Improvement on LEACH protocol of wireless sensor network, in: Proceedings of International Conference on Sensor Technologies and Applications (Sensor Comm), Oct.2007, 260-264, doi:10.1109/SENSORCOMM.2007.4394931.

[17] J. Yu, Y. Qi, G.Wang, X. Gu, A cluster-based routing protocol for wireless sensor networks with non-uniform node distribution, International J. of Electronics and Communications (AEÜ) 66 (2012) 54-61.

[18] J. Yu, Y. Qi, G.Wang, An energy driven unequal clustering protocol for heterogeneous wireless sensor networks, J. Control Theory Appl. 9 (1) (2011) 133-139.

[19] I. Dietrich, F. Dressler, on the lifetime of wireless sensor networks, ACM Transactions on Sensor Networks, 5(1) (2009) 1-38, doi: 10.1145/1464420.1464425.

[20] D. Puccinelli, M. Haenggi, Wireless sensor networks: Applications and challenges of ubiquitous sensing, IEEE Circuits and Systems Magazine, 2005, 19-29.

[21] N.H. Mak, How long is the lifetime of wireless senor network?, in: Proc. IEEE International Conference on Advanced Information Networking and Applications, 2009, 763-770.

[22] X. Wu, G. Chen, S. K. Das, Avoiding energy holes in wireless sensor networks with nonuniform node distribution, IEEE Transactions on Parallel and Distributed Systems 19 (5) (2008) 710-720.

[23] Y.Yang, M. Cardei, Movement-assisted sensor redeployment scheme for network lifetime increase, in: 10th ACM / IEEE Intl. Symposium on Modeling, analysis and simulation of Wireless and Mobile Systems (MSWIM), 2007, pages 13-20, doi:10.1145/1298126. 1298132.

[24] J. Luo and J.P. Hubaux, Joint mobility and routing for lifetime elongation in wireless sensor networks, in: Proc. of IEEE, INFOCOM 2005, pp. 1735-1746.

[25] M. Marta, M. Cardei, Improved sensor network lifetime with multiple mobile sinks 5 (5) (2009) 542 - 555.

[26] H.M. Ammari, S.K. Das, Promoting heterogeneity, mobility, and energy-aware voronoi diagram in wireless sensor networks, IEEE Transactions on Parallel and Distributed Systems 19 (7) (2008) 995-1008. 
[27] D.Vass, Z. Vincze, R. Vida and A. Vidacs, Energy efficiency in wireless sensor networks using mobile base station, in EUNICE 2005: Networks and applications towards a ubiquitously connected world, 2005, 173-186.

[28] J. Li, P. Mohapatra, An analytical model on the energy hole problem in many-to-one sensor networks, in: Proc. of IEEE Vehicular Technology Conf., Fall 2005, pp. 2721 2725.

[29] J. Wu, Y. Qi, G. Wang, Q. Guo, X. Gu, An energy aware distributed unequal clustering protocol for wireless sensor networks, International Journal of Distributed Sensor Networks, 2011, doi:10.1155/2011/202145.

[30] J. Li, P. Mohapatra, Analytical modeling and mitigation techniques for the energy hole problem in sensor networks, Pervasive and Mobile Computing 3 (2007) 233-254.

[31] J. Lian, K. Naik, G. Agnew, Data capacity improvement of wireless sensor networks using non-uniform sensor distribution, International J. of Distributed Sensor Networks 2(2) (2006) 121-145.

[32] J. Jia, X. Wu, J. Chen, X. Wang, Exploiting sensor redistribution for eliminating the energy hole problem in mobile sensor networks, Eurasip J. on Wireless Communications and Networking 1 (2012) 1-11.

[33] L.Qing, Q.Zhu, M.Wang, Design of a distributed energyefficient clustering algorithm for heterogeneous wireless sensor networks, Computer Communications 29 (2006) 2230-2237.

[34] A. F.Liu, W.X. You, C.Z.Gang, G.W. Hua, Research on the energy hole problem based on unequal cluster-radius for wireless sensor networks, Computer Communications 33(2010), 302-321.

[35] H. Shin, S. Moh, H. Chung, M. Kang, Equal-size clustering for irregularly deployed wireless sensor networks, Wireless Personal Communications (2015) 82:995-1012.

[36] T. Agmoth, P.K.Jana, Energy-aware routing algorithm for wireless sensor networks, Computers and Electrical
Engineering, 41(2015) 357-367.

[37] M. Sabet, H.R. Naji, A decentralized energy-efficient hierarchical clustering based routing algorithm for wireless sensor networks, International Journal of Electronics and Communications (AEÜ) 69 (2015) 790799.

\section{Authors' Profiles}

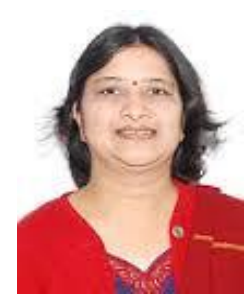

Vrinda Gupta received the B.E. and M.Tech. degree in Electronics and Communication Engineering in 1987, and 1994 from Nagpur University and Kurukshetra University (erstwhile R.E.C. Kurukshetra), India. She is currently pursuing her Ph.D. and is an Associate Professor in the Electronics and Communication Engineering Department at National Institute of Technology, Kurukshetra, India. Her research interests are in the fields of computer networking, and wireless sensor networks.

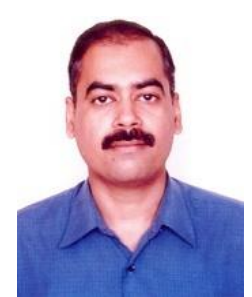

Rajoo Pandey received the B.E., M.Tech., and Ph.D. degrees in Electronics and Communication Engineering in 1989,1991, and 2001 from Government Engineering College, Jabalpur, R.E.C. Kurukshetra, and Indian Institute of Technology, Roorkee, India, respectively. He is now Professor and Head in the Department of Electronics and Communication Engineering, N.I.T. Kurukshetra, India. His research interests include signal and image processing and communication systems.

How to cite this paper: Vrinda Gupta, Rajoo Pandey,"An Improved Energy Aware Distributed Clustering Protocol for Wireless Sensor Networks", International Journal of Computer Network and Information Security(IJCNIS), Vol.8, No.6, pp.29-37, 2016.DOI: 10.5815/ijcnis.2016.06.04 